\title{
A fixed point theorem for weakly inward $A$-proper maps and application to a Picard boundary value problem
}

\section{Casey T Cremins*}

"Correspondence:

ctc@math.umd.edu Department of Mathematics, University of Maryland, College Park, Maryland, USA

\section{Springer}

\begin{abstract}
A fixed point theorem for weakly inward A-proper maps defined on cones in Banach spaces is established using a fixed point index for such maps. The result generalizes a theorem in Deimling (Nonlinear Functional Analysis, 1985) for weakly inward maps defined on a cone in $\mathbb{R}^{n}$. We then apply the theorem to a Picard boundary value problem and obtain the existence of a positive solution.
\end{abstract}

MSC: Primary 34B18; secondary 34B15

Keywords: fixed point index; cone; positive solutions; boundary value problem

\section{Introduction}

The purpose of this paper is to establish a fixed point theorem for weakly inward $A$-proper maps defined on cones in Banach spaces that generalizes a result in Deimling [1], p.254, for weakly inward maps defined on a cone in $\mathbb{R}^{n}$. We use the fixed point index for weakly inward A-proper maps introduced by Lan and Webb [2] to obtain our new result. As an application, we obtain a positive solution to the Picard boundary value problem

$$
-x^{\prime \prime}(t)=f\left(t, x(t), x^{\prime}(t), x^{\prime \prime}(t)\right), \quad \text { where } x(0)=x(1)=0,
$$

under suitable conditions on $f$. This problem has been extensively studied; in particular, we refer to [3], where the concept of $P_{\gamma}$-compact maps and quasinormal cones is used, [4], where the problem is formulated as a semilinear equation, [5], where $f$ is allowed to take negative values, and [6], where positive solutions for three-point boundary value problems are obtained. As mentioned in [5], in [3] and [4], examples were provided with conflicting hypotheses; our theorem will allow a different approach, which corrects the hypotheses of the analogous examples.

\section{Preliminaries}

Let $X$ be a Banach space, $X_{n} \subset X$ a sequence of oriented finite-dimensional subspaces, and $P_{n}: X \rightarrow X_{n}$ a sequence of continuous linear projections such that $P_{n} x \rightarrow x$ for each $x \in X$.

Then $X$ is called a Banach space with projection scheme $\Gamma=\left\{X_{n}, P_{n}\right\}$.

(c) 2016 Cremins. This article is distributed under the terms of the Creative Commons Attribution 4.0 International License (http://creativecommons.org/licenses/by/4.0/), which permits unrestricted use, distribution, and reproduction in any medium, provided you give appropriate credit to the original author(s) and the source, provide a link to the Creative Commons license, and indicate if changes were made. 
A map $f: \operatorname{dom} f \subset X \rightarrow X$ is said to be $A$-proper with respect to $\Gamma$ if $P_{n} f: X_{n} \rightarrow X_{n}$ is continuous for each $n$ and for any bounded sequence $\left\{x_{n_{j}} \mid x_{n_{j}} \in X_{n_{j}}\right\}$ such that $f_{n_{j}}\left(x_{n_{j}}\right) \rightarrow y$, there exists a subsequence $\left\{x_{n_{j_{k}}}\right\}$ such that $x_{n_{j_{k}}} \rightarrow x$ and $f(x)=y$.

A closed convex set $K$ in a Banach space $X$ is called a cone if $\lambda K \subset K$ for all $\lambda \geq 0$ and $K \cap\{-K\}=0$.

Let $K \subset X$ be a closed convex set. For each $x \in K$, the set $I_{K}(x)=\{x+c(z-x): z \in K, c \geq 0\}$ is called the inward set of $x$ with respect to $K$. A map $f: K \rightarrow X$ is called inward (respectively, weakly inward) if for all $x \in K, f(x) \in I_{K}(x)\left(f(x) \in \bar{I}_{K}(x)\right)$.

A map $f: \bar{\Omega}_{K} \rightarrow X$ is said to be inward (respectively, weakly inward) on $\bar{\Omega}_{K}$ relative to $K$ if $f(x) \in I_{K}(x)$ (respectively, $f(x) \in \bar{I}_{K}(x)$ ) for $x \in \bar{\Omega}_{K}$, where $\Omega \subset X$ is open and bounded with $\Omega_{K}=\Omega \cap K \neq \emptyset$.

For the definition and properties of the Lan-Webb fixed point index, see [2].

\section{An existence theorem for weakly inward $A$-proper maps}

Theorem 3.1 Let $K$ be a closed convex set, and $f: K \rightarrow X$ be weakly inward on $K$, where $I-f$ is A-proper. Suppose that

(a) $f(x) \not x$ on $\|x\|=r$, and

(b) there exists $\rho \in(0, r)$ such that $\lambda x \not \leq f(x)$ for $\|x\|=\rho$ and $\lambda>1$.

Then $f$ has a fixed point in $\{x \in K: \rho<\|x\|<r\}$.

Proof Let $B_{r}=\{x \in X:\|x\|<r\}, B_{r_{K}}=B_{r} \cap K, B_{\rho}=\{x \in X:\|x\|<\rho\}$, and $B_{\rho_{K}}=B_{\rho} \cap K$. We show that $i_{K}\left(f, B_{r_{K}}\right)=\{0\}$ and $i_{K}\left(f, B_{\rho_{K}}\right)=\{1\}$, so that by the additivity property of the index $i_{K}\left(f, B_{r_{K}} \backslash B_{\rho_{K}}\right)=i_{K}\left(f, B_{r_{K}}\right)-i_{K}\left(f, B_{\rho_{K}}\right)=\{0\}-\{1\}=\{-1\} \neq\{0\}$, which implies the existence of a fixed point $x \in K$ such that $\rho<\|x\|<r$.

To show that $i_{K}\left(f, B_{r_{K}}\right)=\{0\}$, suppose instead that $i_{K}\left(f, B_{r_{K}}\right) \neq\{0\}$. Then we choose an $a$ with $\|f(x)\| \leq a$ on $\bar{B}_{r_{K}}$ and an $e \in K$ with $\|e\|>r+a$. Define the weakly inward $A$ proper homotopy $H(x, t)=f(x)+t e$. Now if $H(x, t)=x$ for some $(x, t) \in \partial B_{r_{K}} \times[0,1]$, then $f(x)+t e=x$, so that $x \in K$ and $x-f(x)=t e \in K$ so $f(x) \leq x$, which contradicts (a). Thus, $H$ is an admissible homotopy, and $i_{K}\left(H(x, 1), B_{r_{K}}\right)=i_{K}\left(f, B_{r_{K}}\right) \neq\{0\}$. Then there exists $x \in B_{r_{K}}$ with $f(x)+e=x$, so that $\|e\|=\|x-f(x)\| \leq\|x\|+\|f(x)\| \leq r+a$, which contradicts $\|e\|>$ $r+a$. Hence, $i_{K}\left(f, B_{r_{K}}\right)=\{0\}$.

Now we show that $i_{K}\left(f, B_{\rho_{K}}\right)=\{1\}$. Define the weakly inward $A$-proper homotopy $H(x, t)=t f(x)$.

If $H(x, t)=x$ for some $(x, t) \in \partial B_{\rho_{K}} \times[0,1]$, then $t \neq 0$ (this would give $0=x$ on $\partial B_{r_{K}}$ ) and $t f(x)=x$ and $x \in K$, so that $f(x)=\frac{1}{t} x \geq x$, which contradicts (b).

Thus $H(x, t) \neq x$ on $\partial B_{\rho_{K}} \times[0,1]$.

By the homotopy property of the index, $i_{K}\left(H(x, 0), B_{\rho_{K}}\right)=i_{K}\left(H(x, 1), B_{\rho_{K}}\right)=i_{K}\left(f, B_{\rho_{K}}\right)=$ $\{1\}$.

Consequently, $i_{K}\left(f, B_{r_{K}} \backslash B_{\rho_{K}}\right)=i_{K}\left(f, B_{r_{K}}\right)-i_{K}\left(f, B_{\rho_{K}}\right)=\{0\}-\{1\}=\{-1\}$.

Since the index is not 0 , the existence property implies that there exists a fixed point $x \in K$ such that

$$
\rho<\|x\|<r
$$

Remark 3.1 The conclusion of Theorem 3.1 is valid if condition (a) holds for $\|x\|=\rho$ and condition (b) holds for $\|x\|=r$, that is, 
(a) $f(x) \not \leq x$ on $\|x\|=\rho$, and

(b) $\lambda x \not \leq f(x)$ for $\|x\|=r$ and $\lambda>1$.

We shall use these conditions in the following application.

\section{Application}

We formulate the Picard boundary value problem

$$
-x^{\prime \prime}(t)=f\left(t, x(t), x^{\prime}(t), x^{\prime \prime}(t)\right), \quad \text { where } x(0)=x(1)=0
$$

as a fixed point equation of the operator $T: \bar{K}_{r} \rightarrow K, K_{r}=\{x \in K:\|x\|<r\}$,

$$
T y(t)=f\left(t, L^{-1} y, \frac{d}{d t}\left(L^{-1} y\right),-y\right),
$$

where $L: X \rightarrow Y$ is defined by $L x=-x^{\prime \prime}(t)$. Observe that (1) is equivalent to $y=T y$.

Let $X=\left\{x \in C^{2}[0,1]: x(0)=x(1)=0\right\}, Y=C[0,1]$, and $K=\{y \in C[1,0]: y(t) \geq 0\}$ with norms $\|x\|_{X}=\max \left\{\|x\|_{Y},\left\|x^{\prime}\right\|_{Y},\left\|x^{\prime \prime}\right\|_{Y}\right\}$ and $\|x\|_{Y}=\max _{t \in[0,1]}\{|x(t)|\}$. Then $L$ is a linear bounded isometric homeomorphism.

\section{Theorem 4.1 Under the above assumptions, suppose also that}

$\left(\mathrm{a}^{\prime}\right)$ there exist $r>0$ and $k \in(0,1)$ such that $f:[0,1] \times[0, r] \times[-r, r] \times R^{-} \rightarrow R^{+}$is continuous with $\left|f\left(t, p, q, s_{1}\right)-f\left(t, p, q, s_{2}\right)\right| \leq k\left|s_{1}-s_{2}\right|$ for $t \in[0,1], p \in[0, r], q \in[-r, r]$, $s_{1}, s_{2} \in R^{-}$;

(b') $f(t, p, q, s)<r$ for every $t \in[0,1], p \in[0, r], q \in[-r, r], s=-r$;

$\left(\mathrm{c}^{\prime}\right)$ there are $\rho \in(0, r), t_{0} \in[0,1]$ such that $f\left(t_{0}, p, q, s\right)>\rho$ for $p \in[0, \rho], q \in[-\rho, \rho], s=-\rho$.

Then there exists a positive solution $x \in K$ to equation (1) with $\rho<\|x\|_{X}<r$.

Proof Since $T$ maps $K$ to $K, T$ is weakly inward. Condition ( $\left.\mathrm{a}^{\prime}\right)$ implies that $T$ is $\left(\beta_{K}\right) k$-ball contractive, where $\beta_{K}$ is the ball measure of noncompactness associated with $K$, and thus $\lambda I-T$ is $A$-proper with respect to the projection scheme $\Gamma=\left\{X_{n}, P_{n}\right\}$ for every $\lambda \geq \gamma$, $\gamma \in(k, 1)\left(c f\right.$. [3]). To verify the remaining hypotheses of Remark 3.1, we first show that ( $\left.\mathrm{b}^{\prime}\right)$ implies (b). Let $r$ be as in ( $\left.\mathrm{b}^{\prime}\right)$ and $y \in K$ such that $\|y\|_{Y}=r$. Then there exists $x \in L^{-1}(K)$ such that $L x=y$ and $\|x\|_{X}=\|y\|_{Y}=\left\|x^{\prime \prime}\right\|_{Y}$, so that $r=\left\|x^{\prime \prime}\right\|_{Y}=\|x\|_{X}$ and there exists $t_{0} \in$ $[0,1]$ such that $y\left(t_{0}\right)=r$. Now since $y=L x$ for some $x \in L^{-1}(K)$, we have that $x(t) \in[0, r]$, $x^{\prime}(t) \in[-r, r]$ for all $t \in[0,1]$ and $r=-x^{\prime \prime}\left(t_{0}\right)$. Then if $T y=\lambda y$ for some $\lambda>1$ and $y \in K$ with $\|y\|_{Y}=r$, we would have $f\left(t, x(t), x^{\prime}(t), x^{\prime \prime}(t)\right)=\lambda y(t)$ for all $t \in[0,1]$, including $t_{0}$, but then this implies $\lambda r<r$, a contradiction. So (b) holds.

To show that ( $\left.\mathrm{c}^{\prime}\right)$ implies (a) of Remark 3.1, let $x \in K$ with $\|x\|_{X}=\rho$. Then $\|L x\|_{Y}=$ $\left\|-x^{\prime \prime}\right\|_{Y}=\rho$, and there exists $t_{1} \in[0,1]$ such that $-x^{\prime \prime}\left(t_{1}\right)=\rho$ or $x^{\prime \prime}\left(t_{1}\right)=-\rho$. So we have for $t \in[0,1]$ that $x(t) \in[0, \rho], x^{\prime}\left(t_{1}\right) \in[-\rho, \rho]$, and $x^{\prime \prime}\left(t_{1}\right)=-\rho$. By $\left(\mathrm{c}^{\prime}\right)$ we have $T y\left(t_{1}\right)=$ $f\left(t_{1}, x\left(t_{1}\right), x^{\prime}\left(t_{1}\right), x^{\prime \prime}\left(t_{1}\right)\right)>\rho$, and so (a) is satisfied.

Thus, there exists a solution to equation (1) with $x \in K$ and $\rho<\|x\|<r$.

Example 4.1 The function $f\left(t, x, x^{\prime}, x^{\prime \prime}\right)=1+\frac{3}{4} \sin x^{\prime \prime}$ with $r=\frac{3 \pi}{2}$ and $\rho=\frac{\pi}{2}$ shows that the class of maps that satisfy the conditions of Theorem 4.1 is nonempty. 
Competing interests

The author declares that he has no competing interests.

\section{Acknowledgements}

The author is grateful to the referees for their useful comments that have improved this paper.

Received: 22 January 2016 Accepted: 17 June 2016 Published online: 29 June 2016

\section{References}

1. Deimling, K: Nonlinear Functional Analysis. Springer, Berlin (1985)

2. Lan, K, Webb, JRL: A fixed point index for weakly inward A-proper maps. Nonlinear Anal. 28, 315-325 (1997)

3. Lafferriere, B, Petryshyn, WV: New positive fixed point and eigenvalue results for $P_{\gamma}$-compact maps and applications. Nonlinear Anal. 13, 1427-1440 (1989)

4. Cremins, CT: Existence theorems for semilinear equations in cones. J. Math. Anal. Appl. 265, 447-457 (2002)

5. Lan, K, Webb, JRL: A-Properness of contracting and condensing maps. Nonlinear Anal. 49, 885-895 (2002)

6. Infante, G: Positive solutions of some three point boundary value problems via fixed point index for weakly inward A-proper maps. Fixed Point Theory Appl. 2005, 177-184 (2005)

\section{Submit your manuscript to a SpringerOpen ${ }^{\circ}$ journal and benefit from:}

- Convenient online submission

Rigorous peer review

- Immediate publication on acceptance

- Open access: articles freely available online

- High visibility within the field

- Retaining the copyright to your article 